\title{
Aneurismas infecciosos: incidência, diagnóstico e tratamento
}

\author{
Saulo Simões Garciaํ, Eberval Gadelha Figueiredo², Manoel Jacobsen Teixeira ${ }^{3}$ \\ Divisão de Neurocirurgia do Hospital das Clínicas Faculdade de Medicina da Universidade de São Paulo (HC-FMUSP).
}

\section{RESUMO}

O objetivo deste trabalho é apresentar a revisão de literatura acerca dos novos parâmetros de incidência, diagnósticos e terapêuticos, dos aneurismas infecciosos. Foi realizada pesquisa no banco de dados Bireme e PubMed,com os termos: "aneurysm", "mycotic" e "infectious", sendo usados para o estudo apenas artigos publicados entre 1997 e 2010. Grande parte dos artigos descreve uma alta incidência de múltiplos aneurismas e a localização preferencialmente proximal, quando associados à endocardite infecciosa, e distal, quando associados a outras infecções, também relatando a alta eficiência do tratamento endovascular, contudo uma alta mortalidade quando este é inviável. Novas técnicas diagnósticas são propostas e merecem mais estudos. A taxa de incidência e a alta taxa de mortalidade associada a casos em que a intervenção endovascular é inviável justificam exames direcionados ao diagnóstico precoce desses aneurismas em pacientes potenciais.

\section{PALAVRAS-CHAVE}

Aneurisma infectado, endocardite bacteriana, meningite.

\section{ABSTRACT}

The infectious aneurysms: incidence, diagnosis and treatment

The objective of this paper is to present a literature review of incidence, diagnosis and treatment of infectious aneurysms. A search was carried out at the Bireme database and PubMed by the terms: "aneurysm", "mycotic" and "infectious", being used to study only articles published between 1997 and 2010. Most of the articles describe a high incidence of multiple aneurysms, and proximal location when associated with infective endocarditis and distal when associated with other infections. It is reported a high efficiency in endovascular treatment, but a high mortality when unviable. New diagnostic techniques are proposed and deserve further study. The incidence and high mortality rate associated with cases in which endovascular intervention is not feasible, justify early diagnosis of these aneurysms in potential patients.

\section{KEYWORDS}

Aneurysm infected, endocarditis bacterial, meningitis.

\section{Introdução}

Aneurismas infecciosos, previamente chamados de aneurismas micóticos, são formados a partir de um processo tromboembólico, tendo sua etiologia em uma infecção bacteriana, microbacteriana, fúngica ou até mesmo viral. Por ser o aneurisma infeccioso uma complicação secundária à infecção, ele acaba sendo negligenciado durante o diagnóstico e o tratamento da doença, mesmo sendo causa importante do agravamento do quadro clínico.

Este estudo pretende avaliar os diversos aspectos do aneurisma infeccioso, pesquisando, para isso, artigos publicados no período de 1996 a 2010, encontrados por meio de busca no banco de dados da Bireme e PubMed, com os termos "aneurysm", "mycotic" e "infectious" e suas traduções em português.

\section{Revisão de literatura}

Em 2007, Kannoth et al. ${ }^{6}$ analisaram o banco de dados do Sree Chitra Tirunal Institute for Medical Science and Technology, no período de 1976 a 2003, em um

1. Faculdade de Medicina da Universidade do Estado do Pará (UEPA), Belém, PA, Brasil.

2. Faculdade de Medicina da Universidade de São Paulo (FMUSP), Divisão de Clínica Neurocirúrgica do Hospital das Clínicas (HC) da FMUSP, São Paulo, SP, Brasil.

3. Departamento de Neurologia da FMUSP, Divisão de Neurocirurgia Funcional do Instituto de Psiquiatria do HC-FMUSP, São Paulo, SP, Brasil. 
acervo de 1.740 cirurgias de aneurisma intracraniano, distinguindo os de origem infecciosa por meio do diagnóstico de endocardite infecciosa nos pacientes, segundo os critérios de Dukes e os de Thuaites.

Foram encontrados 25 casos, o que corresponde a 1,44\% da amostra total. Em $20 \%$ dos pacientes, o diagnóstico foi dado a partir de autópsia, sendo dos demais pacientes $15 \%$ com sintomatologia clínica e $85 \%$ a partir de achados radiológicos. Foi realizada tomografia computadorizada em 15 casos, sendo encontrada hemorragia em 13 (86,6\%) exames, com mais de um foco hemorrágico em alguns pacientes. A angiografia, realizada em 23 pacientes, detectou aneurisma em 20 casos, e nos outros três foi detectado durante a autópsia. Os aneurismas de origem fúngica tiveram uma localização em artérias proximais em $75 \%$ dos aneurismas de etiologia fúngica, enquanto os de origem infecciosa se localizaram em artérias distais em $90 \%$ dos aneurismas com essa etiologia. Os autores destacam as diferenças na história natural da doença dos aneurismas infecciosos de acordo com sua etiologia, devendo-se levar tal fator em conta quando da escolha do tratamento adequado.

Phuong et al. ${ }^{11}$ publicaram, em 2002, 16 casos de aneurisma infeccioso em Mayo Clinic, Rochester, ocorridos no período de 1976 a 1999, estando no grupo de estudo pacientes com aneurisma diagnosticado via angiografia ou autópsia, e infecção, diagnosticada por meio de cultura sanguínea ou vegetação valvular.

Os sintomas mais comuns apresentados entre os 16 casos foram febre e calafrios $(87,5 \%)$ e cefaleia não específica (43,7\%); seis pacientes $(37,5 \%)$ apresentaram letargia e confusão; três (18,7\%) apresentaram quadros convulsivos e perda da acuidade visual. Foram encontrados 29 aneurismas nos 16 pacientes, tendo cinco pacientes mais de um aneurisma, variando de dois a oito aneurismas em cada. A localização foi anterior em 16 aneurismas e posterior nos 13 restantes. Quatro dos pacientes com apenas um aneurisma não apresentaram hemorragia, portanto não seriam diagnosticados mediante tomografia computadorizada.

Em 2000, Venkatesh et al. ${ }^{13}$ reportaram 17 pacientes diagnosticados com aneurisma infeccioso, atendidos no período de 1989 a 1999. Todos os aneurismas foram confirmados por angiografia, com diagnóstico prévio de endocardite infecciosa em 12 pacientes, sendo posteriormente confirmado nos demais.

Foram encontrados 22 aneurismas nos 17 pacientes: 90,9\% dos aneurismas encontrados tinham localização distal e apenas $9,1 \%$, proximal. Pertenciam à circulação posterior $60 \%$ dos aneurismas. Foram classificados como aneurismas pequenos (3-5 mm) 15 dos 22 aneurismas encontrados, e oito foram classificados como médios (6-9 mm). Apresentaram contornos irregulares $72,7 \%$ do total. Oito dos 10 aneurismas rompidos que tiveram tratamento conservador evoluíram para cura. Receberam tratamento cirúrgico seis aneurismas rompidos, tendo todos bons resultados. Os autores concluíram que, mediante tratamento conservador medicamentoso, os pacientes evoluíram bem, contudo se faz necessário um acompanhamento permanente.

Chun et al. ${ }^{4}$ estudaram a eficiência do tratamento multiprofissional no tratamento de aneurisma infeccioso de 1990 a 2000, tendo uma amostra de 27 aneurismas em 20 pacientes, sendo $65 \%$ dos aneurismas de etiologia na endocardite infecciosa e a principal localização na artéria cerebral média.

Foram tratados por via endovascular cinco dos 20 pacientes, 10 por microcirurgia e o restante de maneira conservadora, fazendo o acompanhamento medicamentoso e da evolução do aneurisma. Os autores relatam que os fatores que determinaram o procedimento a ser tomado com cada paciente foram a ruptura do aneurisma, hematomas que alteraram a pressão intracraniana (PIC) e a oxigenação adequada do tecido cerebral. $\mathrm{O}$ tratamento medicamentoso foi usado em pacientes sem ruptura do aneurisma, e o tratamento endovascular, em pacientes sem hematoma ou em hematomas laminares. O tratamento cirúrgico foi a primeira escolha nos pacientes com hemorragia ou quando houve falha dos demais tratamentos.

No ano 2000, Ruiz et al. ${ }^{12}$ estudaram casos ocorridos de endocardite infecciosa na cidade de Ribeirão Preto SP, analisando a etiologia, a epidemiologia, os aspectos clínicos e a evolução dos quadros clínicos.

O estudo teve uma casuística de 180 casos de endocardite infecciosa, em 168 pacientes, sendo feita hemocultura em 93,8\% dos casos e encontrados Staphylococcus aureus em 27,2\% e Streptococcus viridans em 15,9\% dos pacientes. Dentre os pacientes, $40,5 \%$ faleceram e $64,4 \%$ tiveram alguma complicação, sendo $21,5 \%$ dessas complicações aneurismas infecciosos. Conclui-se que há grande incidência de aneurismas como complicação de endocardite infecciosa, acarretando o elevado índice de mortalidade pela doença.

Aoun et al. ${ }^{1}$ investigaram os fatores de risco de mortalidade intra-hospitalar em adolescentes acometidos com endocardite infecciosa, analisando, para isso, 33 casos de forma retrospectiva admitidos no Hospital Universitário Pedro Ernesto.

Dentre os 17 casos que evoluíram com complicações resultantes em aneurismas infecciosos, $10(58,82 \%)$ faleceram, enquanto entre os 16 pacientes que não apresentaram tal complicação a mortalidade foi de $25 \%$ (quatro pacientes), sendo essa diferença marginalmente significativa, segundo o autor. Destacou o autor, a partir desse dado, a gravidade de um paciente que evolui com aneurismas em casos de endocardite infecciosa, sendo 
relevante a investigação de aneurismas no decorrer do tratamento.

Em 2004, Barbosa ${ }^{2}$ pesquisou o perfil clínico dos pacientes com endocardite infecciosa na literatura médica, ressaltando a maior incidência da doença em homens (1.7:1) e a mudança no perfil etário acometido pela enfermidade, 47-69 anos atualmente. Ressaltou também a alta incidência da infecção relacionada a alterações valvares degenerativas e próteses valvares e apontou os Staphylococcus aureus como principal etiologia da endocardite infecciosa.

A autora cita que vegetações maiores que $10 \mathrm{~mm}$ são associadas a maior índice de acidente vascular cerebral, mas não a embolias periféricas, o que corrobora a importância da vigilância em relação a aneurismas como agravamento em quadros de endocardite infecciosa.

Wajnberg ${ }^{14}$, em sua tese de mestrado no ano de 2010, dissertou sobre o tratamento endovascular de aneurismas infecciosos cerebrais, estudando, para isso, cinco pacientes atendidos no Hospital Universitário Fraga Filho - UFRJ, no período de 2002-2006, com diagnóstico de endocardite infecciosa, segundo os critérios de Dukes.

O tratamento preferencial foi com a utilização de cianoacrilato, utilizado em três pacientes $(60 \%)$, ocluindo tanto aneurisma quanto seu vaso parental. Foi utilizada nos demais casos (40\%) embolização por micromolas. Dois pacientes faleceram, sendo um por complicações da endocardite. O autor relata que a escolha da técnica usada foi devida à morfologia do aneurisma; foram utilizados cianoacrilatos em aneurismas sem colo, adequados à utilização das micromolas, não tendo nenhuma intercorrência na utilização do produto em um local potencialmente infectado.

Liphaus et al. ${ }^{9}$ estudaram aneurismas infecciosos ocorridos em crianças, analisando um caso, literatura publicada e o banco de dados do Ministério da Saúde brasileiro.

A autora relata que a taxa de incidência dos aneurismas infecciosos chega a $40 \%$ das endocardites infecciosas, tendo a mortalidade nesses casos taxas de até $58 \%$. Avalia também que os sintomas do aneurisma muitas vezes precedem os da endocardite, sendo esse o passo para a descoberta da doença em $47 \%$ dos casos. $\mathrm{O}$ acometimento da artéria mesentérica superior foi o mais relacionado à etiologia infecciosa, sendo de 70\%-90\% desses casos sintomáticos; o de aorta e o de mesentérica superior são os mais comuns na literatura.

Chapot et al. ${ }^{3}$, em 2001, avaliaram o tratamento endovascular em aneurismas infecciosos, analisando, para isso, 14 pacientes tratados por via endovenosa, sendo encontrados 18 aneurismas, localizando-se na circulação distal 13 deles e 5 no polígono de Willis.
Aneurismas distais e fusiformes foram tratados com a oclusão do aneurisma e dos vasos de origem. Todos os pacientes obtiveram êxito no tratamento, sendo apenas dois acompanhados por complicações transitórias (agravamento da hemiparesia e quadrantopsia). A partir da pesquisa, os autores concluíram que o tratamento endovascular para os aneurismas infecciosos é plenamente eficaz.

Braun et al..$^{5}$ avaliaram de maneira multidisciplinar 261 casos de endocardite infecciosa ocorridos entre 1980 e 1990, comparando os diversos aspectos, a evolução em cada tratamento e as complicações encontradas.

Evoluíram com acometimento trombótico causando aneurismas $34 \%$ dos pacientes da amostra, sendo o $S$. aureus associado a maior incidência de êmbolos, levando a complicações que frequentemente contraindicavam a cirurgia; $8 \%$ dos pacientes tinham indicação cirúrgica, mas não a fizeram pelo alto risco associado às complicações, aumentando o índice de mortalidade de tais pacientes em $27 \%$. Concluíram os autores que o tratamento multidisciplinar aumenta a probabilidade de um melhor e mais rápido diagnóstico, influindo de maneira positiva no prognóstico dos pacientes.

Kannoth et al. ${ }^{8}$ propuseram um novo critério diagnóstico para os aneurismas infecciosos em 2008, estudando, para isso, 25 pacientes com aneurisma infeccioso confirmado e 111 pacientes com aneurisma não infeccioso.

O critério se baseia em uma premissa mandatória: a confirmação da existência do aneurisma por meio de diagnósticos por angiografia, associando-se a esses três grupos de agravamentos: 1 - Presença/história recente de infecção predisponente (podendo ser esta endocardite infecciosa, meningite, celulite orbital, tromboflebite do seio cavernoso). 2 - Achados angiográficos (sendo estes aneurismas múltiplos, de localização distal, fusiformes ou com localização diferente em sucessivas angiografias). 3 - Outras características agravantes (idade menor que 45 anos, febre recente ou que perdure por sete ou mais dias, punção lombar recente, hemorragia intraparenquimatosa confirmada por método radiológico). Pontua-se cada critério do grupo de agravamento que contenha uma ou mais respostas afirmativas com um ponto, classificando como clinicamente definitivos pacientes com a premissa mandatória e três pontos nos grupos de agravamento, clinicamente prováveis pacientes com a premissa mandatória e dois pontos nos grupos de agravamento e clinicamente possíveis pacientes com a premissa mandatória e um ponto nos grupos de agravamento. Concluíram os autores que, usando tais técnicas, foi obtido êxito em casos em que o paciente preenche dois ou três critérios dos grupos de agravamento, apresentando uma assertiva de 100\% e $87,4 \%$, respectivamente. 
Merritt - Tratado de Neurologia, editado por Rowland ${ }^{10}$ e publicado no ano de 2005, refere-se aos aneurismas infecciosos como sendo aneurismas causados por êmbolos sépticos mais comumente formados por endocardite infecciosa.

Os aneurismas infecciosos têm a tendência a ter apenas alguns milímetros e ocorrer em ramos distais dos vasos piais, especialmente os da artéria cerebral média. Relatando a presença de aneurismas infecciosos em até $10 \%$ dos pacientes com endocardite infecciosa, o autor alerta sobre a não periodicidade da realização da arteriografia em busca de tal aneurisma, levando em conta que a mortalidade por rompimento de tais aneurismas é de aproximadamente $80 \%$, sendo o prognóstico de difícil previsão e a necessidade do tratamento o quanto antes possível.

Em 2009, Kannoth e Thomas ${ }^{7}$ pesquisaram as novas discussões acerca do diagnóstico e tratamento do aneurisma infeccioso, termo que o próprio autor trata como mais adequado por serem múltiplas as possíveis causas da enfermidade.

Os autores apontam como características dos aneurismas infecciosos a incidência de múltiplos aneurismas em um mesmo paciente e o fato de eles serem distais e fusiformes, contudo aponta uma apresentação clínica e radiológica variável. Sendo a hemorragia intracraniana a forma mais frequente de apresentação desses aneurismas, exames de angiografia são a melhor forma diagnóstica. Os aneurismas infecciosos têm taxa de mortalidade maior quando rompidos em comparação aos aneurismas não infecciosos, sendo o tratamento conservador (antibióticos), cirurgia ou tratamento endovascular, contudo a indicação de antibióticos deve ser feita em todos os casos. Os autores relataram ser o tratamento da infecção subjacente parte importante da terapia.

\section{Conflitos de interesses}

Os autores declaram não haver conflitos de interesse científico neste estudo.

\section{Referências}

1. Aoun NB, Albanesi Filho FM, Rachid MB, Fernandes LA, Messias JA. Endocardite infecciosa em adolescentes. Análise dos fatores de risco de mortalidade intra-hospitalar. Arq Bras Cardiol. 1997;69(6):407-12.

2. Barbosa MM. Endocardite infecciosa: perfil clínico em evolução. Arq Bras Cardiol. 2004;83(3):189-90.

3. Chapot R, Houdart E, Saint-Maurice JP, Aymard A Mounayer C, Lot G, etal. Endovascular treatment of cerebral mycotic aneurysms. Radiology. 2002;222(2):389-96.

4. Chun JY, Smith W, Halbach VV, Higashida RT, Wilson CB, Lawton MT. Current multimodality management of infectious intracranial aneurysms. Neurosurgery. 200;48(6):1203-13.

5. Braun S, Escalona A, Chamorro G, Corbalán R, Pérez C, Labarca J, et al. Infective endocarditis: short and longterm results in 261 cases managed by a multidisciplinary approach. Rev Med Chil. 2000;128(7):708-20.

6. Kannoth S, Iyer R, Thomas SV, Furtado SV, Rajesh BJ, Kesavadas $C$, et al. Intracranial infectious aneurysm: presentation, management and outcome. J Neurol Sci. 2007;256(1-2):3-9.

7. Kannoth S, Thomas SV. Intracranial microbial aneurysm (infectious aneurysm): current options for diagnosis and management. Neurocrit Care. 2009;11(1):120-9.

8. Kannoth S, Thomas SV, Nair S, Sarma PS. Proposed diagnostic criteria for intracranial infectious aneurysms. J Neurol Neurosurg Psychiatry. 2008;79(8):943-6.

9. Liphaus BL, Goldenstein-Schainberg C, Kitamura LM, Silva CA. Aneurisma micótico abdominal e embolia cerebral associados à endocardite infecciosa em paciente com doença valvar reumática crônica. Arq Bras Cardiol. 2007;88(1):e7-9.

10. Mayer AS, Bernardini GL, Solomon RA, Brust JC Hemorragia subaracnóidea. In: Rowland LP, editor. Merritt - Tratado de Neurologia. 5. ed. Rio de Janeiro: Guanabara Koogan; 2005. p.313.

11. Phuong LK, Link M, Wijdicks E. Management of intracranial infectious aneurysms: a series of 16 cases. Neurosurgery. 2002;51(5):1145-51.

12. Ruiz Jr E, Schirmbeck T, Figueiredo LT. Estudo sobre endocardite infecciosa em Ribeirão Preto, SP - Brasil. Análise de casos ocorridos entre 1992 e 1997. Arq Bras Cardiol. 2000;74(3):217-31.

13. Venkatesh SK, Phadke RV, Kalode RR, Kumar S, Jain VK. Intracranial infective aneurysms presenting with haemorrhage: an analysis of angiographic findings, management and outcome. Clin Radiol. 2000;55(12):946-53.

14. Wajnberg E. Tratamento endovascular de aneurismas infecciosos cerebrais. Radiol Bras. 2010;43(3):210.

Endereço para correspondência

Saulo S. Garcia

Av. Gentil Bittencourt, 2527, São Braz

66063-090 - Belém, PA

Telefone: (91) 8197-1281

E-mail: saulosgarcia@gmail.com 